\title{
Effectiveness of erenumab and onabotulinumtoxinA on acute medication usage and health care resource utilization as migraine prevention in the United States
}

\author{
Stewart J Tepper, MD; Juanzhi Fang, MD, MS; Lujia Zhou, MPH; Ying Shen, MPH; Pamela Vo, PharmD, MS; \\ Ahmad Abdrabboh, PharmD, MPH, BCPS; Mrudula B Glassberg, PhD; and Matias Ferraris, DVM, MBA
}

\section{What is already known about this subject}

- Migraine is a common neurological disease that affects approximately 39 million people in the United States.

- The use of acute medication in the treatment of migraine is often associated with limited effectiveness and poor tolerability, while the overuse of acute medication can lead to disease progression, adverse comorbid medical consequences, and the development of medication overuse headache.

- The health care resource utilization and cost associated with migraine impose a substantial economic burden on the United States.

\section{What this study adds}

- This study provides comparative effectiveness data on the impact of erenumab and onabotulinumtoxinA as migraine preventive therapies on acute medication usage and health care resource utilization.

- Erenumab reduced acute medication usage (opioids and nonsteroidal anti-inflammatory drugs; any acute medication when analyzed together) and health care resource utilization to a greater extent than onabotulinumtoxinA in the 6-month post-index period.

- Erenumab treatment may be associated with a lower frequency of migraine attacks compared with onabotulinumtoxinA as assessed using a composite endpoint as a proxy to evaluate migraine attacks.

OBJECTIVE: To evaluate the real-world impact of erenumab and onabotulinumtoxinA on acute medication usage and health care resource utilization (HCRU) among patients with migraine in the United States.

METHODS: This retrospective US claims analysis (Optum's deidentified Clinformatics Data Mart Database) evaluated patients aged at least 18 years diagnosed with migraine who initiated erenumab or onabotulinumtoxinA between May 1, 2018, and September 30, 2019 (index date: first erenumab/onabotulinumtoxinA claim). Cohorts were matched $1: 1$ using the propensity score

\author{
Author affiliations \\ Stewart J Tepper, MD, Geisel School of \\ Medicine at Dartmouth, Hanover, $\mathrm{NH}$. \\ Juanzhi Fang, MD, MS; Ahmad Abdrabboh, \\ PharmD, MPH, BCPS; and Mrudula B \\ Glassberg, PhD, Novartis Pharmaceuticals \\ Corporation, East Hanover, NJ. Lujia Zhou, \\ $\mathrm{MPH}$, and Ying Shen, $\mathrm{MPH}, \mathrm{KMK}$ Consulting \\ Inc., Morristown, NJ. Pamela Vo, PharmD, \\ MS, and Matias Ferraris, DVM, MBA, Novartis \\ Pharma AG, Basel, CH-4002, Switzerland. \\ AUTHOR CORRESPONDENCE: \\ Juanzhi Fang, 862.754.1752; \\ jenny.fang@novartis.com \\ J Manag Care Spec Pharm. \\ 2021;27(9):1157-70 \\ Copyright $\odot 2021$, Academy of Managed \\ Care Pharmacy. All rights reserved.
}

(PS) method (greedy match with caliper $=0.1$ ). Stratification was performed based on gender, chronic migraine without aura diagnosis, onabotulinumtoxinA use, and acute/ preventive drug use. The impact of erenumab and onabotulinumtoxinA on acute medication usage and HCRU was assessed in the 6-month post-index period. An exploratory analysis assessed the impact of erenumab and onabotulinumtoxinA on a composite endpoint of: (1) outpatient visit with a migraine diagnosis and associated acute medication claim, (2) hospital admission with a primary migraine diagnosis, or (3) emergency 
department visit with a primary migraine diagnosis. PS-matched data were used for comparative analyses; logistic regression with covariate adjustment was used for dichotomous variables, and a negative binomial model was used for count variables, with odds ratios or rate ratios (RRs) and $95 \%$ Cls calculated.

RESULTS: Following stratified PS matching, 1,338 patients were included in both cohorts. At 6 months, the adjusted average number of claims per person for any acute medication was significantly lower in the erenumab cohort (1.13 vs 1.29 in the onabotulinumtoxinA cohort; $\mathrm{RR}=0.88 ; 95 \% \mathrm{Cl}=0.80-0.96 ; P=0.0069$ ), although the difference in the number of claims for triptans and barbiturates was statistically nonsignificant. The adjusted average number of all-cause and migraine-specific visits per person to health care providers was generally lower in the erenumab cohort compared with the onabotulinumtoxinA cohort. Patients in the erenumab cohort had a significantly lower number of composite events ( 0.44 vs 0.69 in the onabotulinumtoxinA cohort; $\mathrm{RR}=0.63 ; 95 \% \mathrm{Cl}=0.56-0.71$; $P<0.0001)$. Similarly, the adjusted proportion of patients with any of the 3 composite events was lower in the erenumab cohort $(31.7 \%$ vs $44.3 \%$ in the onabotulinumtoxinA cohort; $\mathrm{OR}=0.59 ; 95 \% \mathrm{Cl}=0.49-0.70$; $\mathrm{P}<0.0001)$

CONCLUSIONS: In this retrospective claims analysis study, erenumab significantly reduced acute medication usage (opioids and nonsteroidal anti-inflammatory drugs; any acute medication when analyzed together) and HCRU to a greater extent than onabotulinumtoxinA.

Migraine is a common, debilitating neurological disease that affects approximately 39 million people in the United States and 1 billion people worldwide. ${ }^{1}$ Depending on the frequency and regularity of symptoms, migraine can either be categorized as episodic migraine (EM), defined as fewer than 15 headache-days per month, or chronic migraine (CM), defined as at least 15 headache-days per month for more than 3 months, of which at least 8 days have features of migraine. ${ }^{2}$ Migraine imposes a significant burden on patients in terms of pain, disability, reduced quality of life, and reduced work productivity, and it consistently ranks among the top 10 leading causes of years lived with disability worldwide. ${ }^{3}$ Migraine also imposes a heavy economic burden, with the majority of direct costs due to greater utilization of health care resources compared with matched individuals without migraine. . $^{4-6}$

Pharmacological treatment of migraine involves both acute and preventive therapy, with their use depending on the severity and frequency of attacks, as well as patient characteristics and preferences. ${ }^{7-9}$ Acute treatment consists of both migraine-specific medication, such as triptans and ergots, and more recently, lasmiditan and gepants, as well as nonspecific medication, such as nonsteroidal anti-inflammatory drugs (NSAIDs). Opioids and barbiturate-containing medications are not recommended for the treatment of migraine but are still frequently prescribed.$^{10}$ The ultimate goal of acute treatment is to provide a sustained pain-free response upon onset of an attack. ${ }^{9}$ However, acute treatments are often associated with limited effectiveness and poor tolerability, ${ }^{11,12}$ and many of these medications have certain contraindications in different patient subgroups. ${ }^{8}$ Overuse of acute medication is also a well acknowledged problem in migraine management ${ }^{13-15}$ and is associated with the transition from EM to $\mathrm{CM}$, with greater pain intensity and with the development of medication overuse headache $(\mathrm{MOH}) .^{16-18}$

In contrast, the goal of preventive treatment is to reduce the frequency, severity, and duration of attacks in order to improve patient quality of life, as well as to reduce the excessive use of acute treatment., ${ }^{7,819}$ Preventive treatment has typically involved the use of medications such as beta blockers, anticonvulsants, and antidepressants that were originally developed for other conditions and subsequently repurposed for migraine. ${ }^{20,21}$ A substantial number of patients discontinue such treatment due to lack of efficacy and/or poor tolerability. ${ }^{22-24}$

In October 2010, the US Food and Drug Administration (FDA) approved onabotulinumtoxinA (Botox) injection therapy for the preventive treatment of $\mathrm{CM},{ }^{25-28}$ making it the only therapy approved by the FDA specifically for that indication. Since 2018, the FDA has approved 4 monoclonal antibody $(\mathrm{mAb})$ drugs that target the calcitonin gene-related peptide (CGRP) pathway (erenumab, fremanezumab, galcanezumab, and eptinezumab), which were purposely designed for the prevention of migraine. ${ }^{29-37}$ Of those, erenumab (erenumabaooe in the US; Aimovig) is the first and only fully human mAb designed to specifically block the CGRP receptor, which plays a key role in migraine pathophysiology. ${ }^{38}$ The clinical efficacy and safety of erenumab were established in several placebo-controlled studies in $\mathrm{EM}$ and $\mathrm{CM}^{33,34,39,40 \text {; }}$ however, the real-world comparative effectiveness of erenumab has not been fully investigated.

The aim of this study was to evaluate the effectiveness of erenumab and onabotulinumtoxinA in a real-world setting by examining acute medication usage and health care resource utilization (HCRU) among commercially insured patients with migraine using a US administrative claims database. 


\section{Methods}

\section{DATA SOURCE}

Data were extracted from Optum's deidentified Clinformatics Data Mart (CDM) Database, a database of administrative health claims of beneficiaries from commercial and Medicare Advantage health plans. Data are derived from claims submitted by providers and pharmacies to obtain payment for health care services rendered, track plan membership for premium billing, and track participating physicians who have contracts with health plans to provide services. Such data provide a key source of information for a variety of research efforts, including research related to health care costs and resource utilization, as well as quality and effectiveness.

\section{STUDY DESIGN}

This study was a retrospective, exploratory, treatment effectiveness, noninterventional claims database analysis that aimed to evaluate the real-world impact of erenumab and onabotulinumtoxinA on acute medication usage and HCRU (including all-cause and migraine-specific emergency department [ED]/inpatient visits, office visits, neurologist or headache specialist visits, and other outpatient visits) among patients with migraine 6 months after treatment initiation.

This study was conducted in accordance with the Guidelines for Good Pharmacoepidemiology Practices of the International Society for Pharmacoepidemiology 2016, the Strengthening the Reporting of Observational Studies in Epidemiology guidelines, ${ }^{41}$ and the ethical principles laid down in the Declaration of Helsinki. This study was exempt from institutional review board approval, since only deidentified patient records were used.

\section{STUDY POPULATION}

Eligible patients were selected based on having at least 1 migraine diagnosis between May 1, 2017, and September 30,2019 ; having at least 1 prescription for erenumab or onabotulinumtoxinA between May 1, 2018, and September 30, 2019; having continuous medical/pharmacy coverage in the 12-month pre-index and 6-month post-index periods; and being aged at least 18 years as of the index date.

In the erenumab cohort, patients were required to have at least 3 claims of erenumab in the 6 -month post-index period, including the index date. OnabotulinumtoxinA has a quarterly dosing schedule, and therefore patients were required to have at least 1 prescription for onabotulinumtoxinA and an associated migraine diagnosis on or before 14 days of the medication claim. The index date refers to the first erenumab/onabotulinumtoxinA claim.
Patients were excluded from the erenumab cohort if they used other anti-CGRP therapies during the 12-month pre-index or 6-month post-index period. Patients were excluded from the onabotulinumtoxinA cohort if they had a prescription for onabotulinumtoxinA for any reason or if they used any anti-CGRP therapy, including erenumab, in the 12-month pre-index period, or if they used any anti-CGRP therapy, including erenumab, in the 6-month post-index period.

Data from the 12-month pre-index period for both the erenumab and onabotulinumtoxinA cohorts were used to determine most baseline characteristics (eg, diagnosis of CM without aura, selected comorbidities, preventive medication use). Data from the 6-month pre-index period were used to determine baseline acute medication use.

Since some of the acute and preventive medications are nonmigraine-specific and approved for other conditions, a claim associated with a migraine diagnosis was required. Use of nonmigraine-specific acute medication (NSAIDs, opioids, and barbiturates) required a migraine diagnosis on or before 7 days of the medication claim. Use of nonmigraine-specific preventive medication (eg, anticonvulsants, antidepressants, beta blockers) required a migraine diagnosis on or before 14 days of the medication claim, with a supply of at least 28 days.

\section{OUTCOMES}

In order to evaluate the effectiveness and real-world impact of erenumab and onabotulinumtoxinA on acute medication usage, the number of claims per person for acute medication at a specific class level (triptans, opioids, NSAIDs, ergots, and barbiturates), the proportion of patients who used acute medication, and the number of type of acute medications used in the 6-month post-index period were assessed.

In order to evaluate the effectiveness and real-world impact of erenumab and onabotulinumtoxinA on HCRU, the number of all-cause and migraine-specific ED/inpatient visits, office visits, neurologist or headache specialist visits, and other outpatient visits per person at 6 months following treatment initiation of erenumab or onabotulinumtoxinA were assessed. The proportion of patients with all-cause and migraine-specific visits at 6 months following treatment initiation of erenumab or onabotulinumtoxinA were also evaluated. Diagnosis positions 1-5 were used for migraine-specific office visits, including neurologist or headache specialist visits and other outpatient visits. Diagnosis position 1 (primary diagnosis) was used for migraine-specific ED/inpatient visits.

An exploratory objective was to explore the composite endpoint of: (1) outpatient visits with a diagnosis of migraine 


\section{FIGURE 1 Patient Identification Flowchart}

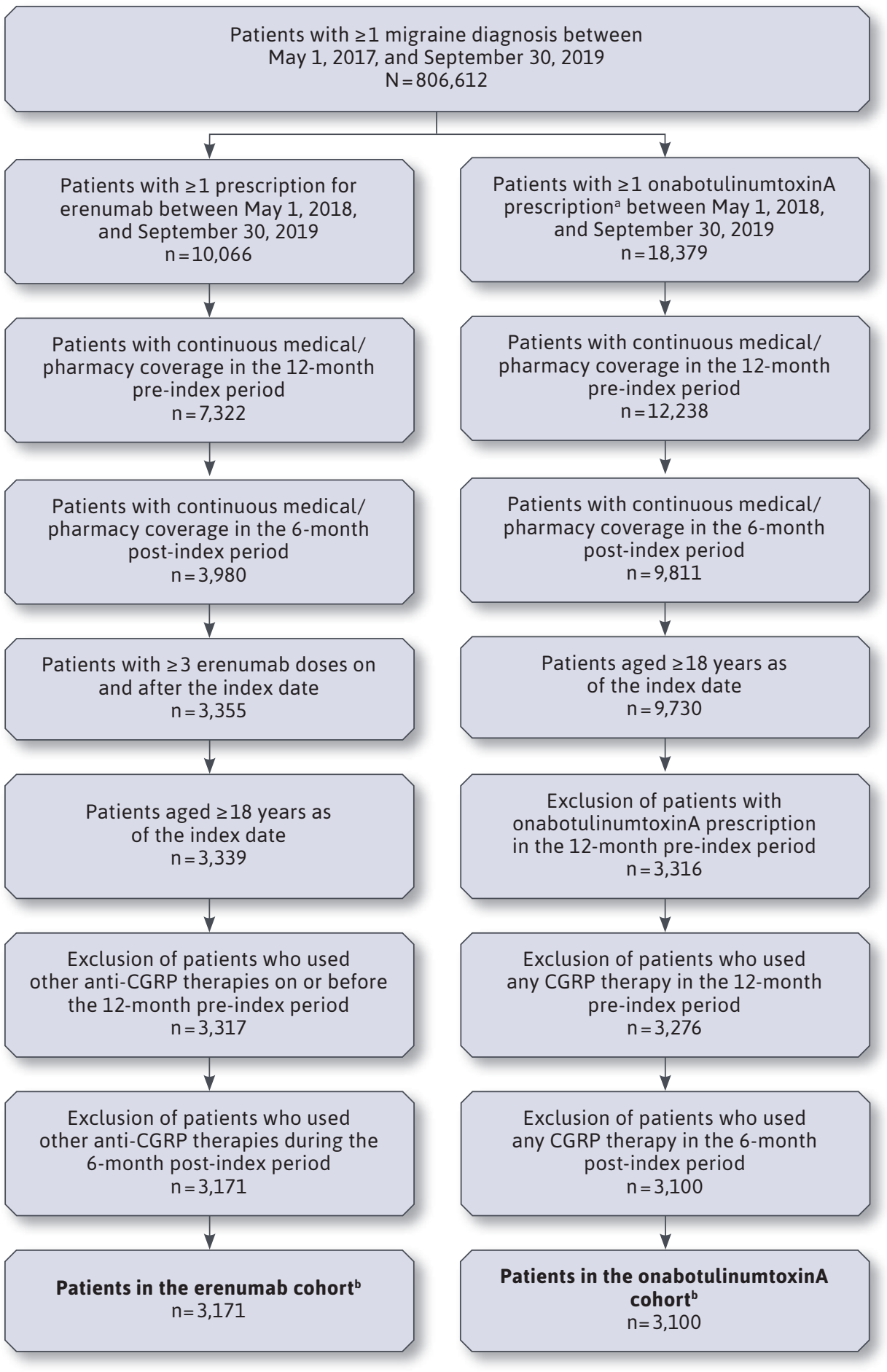

${ }^{a}$ Associated with a migraine diagnosis on or fewer than 14 days following the first migraine diagnosis between May 1, 2018, and September 30, 2019.

${ }^{b}$ Prematched data $(N=1,338$ in both cohorts for postmatched data).

$C G R P=$ calcitonin gene-related peptide and an associated acute medication claim, (2) hospital admissions with a primary diagnosis for migraine, or (3) ED visits with a primary diagnosis for migraine. Any events that occurred on or fewer than 3 days apart were counted only once.

\section{STATISTICAL ANALYSIS}

Data analyses were performed using SAS 3.8 (SAS Institute). Erenumab and onabotulinumtoxinA cohorts were matched 1:1 using the propensity score (PS) method with stratification. Greedy nearest neighbor matching with caliper $=0.1$ was used. Stratification was performed based on gender, diagnosis of $\mathrm{CM}$ without aura, onabotulinumtoxinA use, number of preventive drugs used during the 12-month pre-index period, and number of acute drugs used during the 6-month pre-index period. The variables for PS matching included age, gender, insurance type, region, Charlson Comorbidity Index (CCI), selected comorbidities, acute/preventive drug use, and HCRU. Bivariate analyses of baseline characteristics on pre- and post-PS matching were performed, with the standardized mean difference (SMD) reported to assess the heterogeneity of the 2 cohorts.

PS-matched data were used to assess the comparative effectiveness of erenumab and onabotulinumtoxinA; logistic regression with covariate adjustment was used for dichotomous variables, and a negative binomial model with covariate adjustment was used for count variables, with odds ratios (ORs) or rate ratios (RRs) and 95\% CIs calculated. A proportional odds model was used for ordinal variables. Sensitivity analysis was performed with the inverse probability of treatment weighting (IPTW) model using pre-matched data (Supplementary Materials, available in 


\section{TABLE 1 Demographics and Baseline Clinical Characteristics of Study Patients}

\begin{tabular}{|c|c|c|c|c|c|c|}
\hline \multirow{3}{*}{$\begin{array}{l}\mathbf{n}(\%) \\
\text { Age at index, mean (SD) }\end{array}$} & \multicolumn{3}{|c|}{ Before Matching } & \multicolumn{3}{|c|}{ After Matching } \\
\hline & $\begin{array}{c}\text { Erenumab } \\
n=3,171\end{array}$ & $\begin{array}{l}\text { OnabotA } \\
n=3,100\end{array}$ & \multirow{2}{*}{$\begin{array}{r}\text { SMD } \\
0.07\end{array}$} & $\begin{array}{c}\text { Erenumab } \\
n=1,338\end{array}$ & $\begin{array}{l}\text { OnabotA } \\
n=1,338\end{array}$ & \multirow{2}{*}{$\begin{array}{r}\text { SMD } \\
0.01\end{array}$} \\
\hline & $50.7(13.6)$ & $49.8(14.7)$ & & $50.0 \quad(14.2)$ & $50.2(14.3)$ & \\
\hline Female & $2,689 \quad(84.8)$ & $2,679 \quad(86.4)$ & 0.05 & $1,226 \quad(91.6)$ & $1,226 \quad(91.6)$ & 0.00 \\
\hline CM without aura, 12 -month pre-index period & $1,982 \quad(62.5)$ & $2,202(71.0)$ & 0.18 & $868(64.9)$ & $868 \quad(64.9)$ & 0.00 \\
\hline \multicolumn{7}{|l|}{ Index physician specialty } \\
\hline Neurologist/headache specialist & $2,160 \quad(68.1)$ & $915(29.5)$ & 0.84 & $921(68.8)$ & $410(30.6)$ & 0.83 \\
\hline General practitioner & $442(13.8)$ & $479(15.5)$ & 0.04 & $180(13.5)$ & $203(15.2)$ & 0.05 \\
\hline Nurse/physician assistant & $293 \quad(9.2)$ & $78 \quad(2.5)$ & 0.29 & $122 \quad(9.1)$ & $21 \quad(1.6)$ & 0.34 \\
\hline Unknown/missing & $150 \quad(4.7)$ & $96 \quad(3.1)$ & 0.08 & $60 \quad(4.5)$ & $38 \quad(2.8)$ & 0.09 \\
\hline Other specialist & $58 \quad(1.9)$ & $77 \quad(2.5)$ & 0.05 & $26 \quad(1.9)$ & $37 \quad(2.8)$ & 0.05 \\
\hline Other HCP & $52 \quad(1.6)$ & $1,452 \quad(46.8)$ & 1.24 & $25 \quad(1.9)$ & $628 \quad(46.9)$ & 1.23 \\
\hline Psychiatrist/psychologist & $16 \quad(0.5)$ & $3 \quad(0.1)$ & 0.07 & $4 \quad(0.3)$ & $1 \quad(0.1)$ & 0.05 \\
\hline \multicolumn{7}{|l|}{ Region } \\
\hline South & $1,536 \quad(48.4)$ & $1,219 \quad(39.3)$ & 0.18 & $631 \quad(47.2)$ & $567 \quad(42.4)$ & 0.10 \\
\hline West & $684(21.6)$ & $929(30.0)$ & 0.19 & $291(21.8)$ & $355(26.5)$ & 0.11 \\
\hline Midwest & $667(21.0)$ & $658(21.2)$ & 0.00 & $291(21.8)$ & $299(22.4)$ & 0.01 \\
\hline Northeast & $284 \quad(9.0)$ & $292 \quad(9.4)$ & 0.02 & $125 \quad(9.3)$ & $116 \quad(8.7)$ & 0.02 \\
\hline Unknown & 0 & $2 \quad(0.1)$ & 0.04 & 0 & $1 \quad(0.1)$ & 0.04 \\
\hline \multicolumn{7}{|l|}{ Insurance type } \\
\hline Point of service & $1,569 \quad(49.5)$ & $1,617 \quad(52.2)$ & 0.05 & $666(49.8)$ & $715 \quad(53.4)$ & 0.07 \\
\hline Other & $741(23.4)$ & $576(18.6)$ & 0.12 & $291(21.7)$ & $280(20.9)$ & 0.02 \\
\hline Health maintenance organization & $518(16.3)$ & $490(15.8)$ & 0.01 & $217(16.2)$ & $208(15.6)$ & 0.02 \\
\hline Exclusive provider organization & $214 \quad(6.7)$ & $290 \quad(9.4)$ & 0.10 & $109 \quad(8.2)$ & $86 \quad(6.4)$ & 0.07 \\
\hline Preferred provider organization & $129 \quad(4.1)$ & $127 \quad(4.1)$ & 0.00 & $55 \quad(4.1)$ & $49 \quad(3.7)$ & 0.02 \\
\hline \multicolumn{7}{|c|}{ Selected comorbidities, 12 -month pre-index period ( $>10 \%$ ) } \\
\hline Anxiety & $1,304 \quad(41.1)$ & $1,367(44.1)$ & 0.06 & $536(40.1)$ & $568 \quad(42.5)$ & 0.05 \\
\hline Depression & $1,295 \quad(40.8)$ & $1,312 \quad(42.3)$ & 0.03 & $535(40.0)$ & $557 \quad(41.6)$ & 0.03 \\
\hline Cardiovascular disease & $1,298 \quad(40.9)$ & $1,206 \quad(38.9)$ & 0.04 & $522(39.0)$ & $498 \quad(37.2)$ & 0.04 \\
\hline Insomnia & 731 (23.1) & $675(21.8)$ & 0.03 & $299(22.4)$ & $289(21.6)$ & 0.02 \\
\hline Obesity & $601 \quad(19.0)$ & $530 \quad(17.1)$ & 0.05 & $249(18.6)$ & $219(16.4)$ & 0.06 \\
\hline Fibromyalgia & $434(13.7)$ & $459(14.8)$ & 0.03 & $189(14.1)$ & $202(15.1)$ & 0.03 \\
\hline Constipation & $421 \quad(13.3)$ & 405 (13.1) & 0.01 & $174(13.0)$ & $177(13.2)$ & 0.01 \\
\hline \multicolumn{7}{|c|}{ Number of preventive drug classes used, 12 -month pre-index } \\
\hline 0 & $902(28.5)$ & $1,321 \quad(42.6)$ & 0.30 & $555(41.5)$ & $555(41.5)$ & 0.00 \\
\hline 1 & $1,110(35.0)$ & $991(32.0)$ & 0.06 & $495 \quad(37.0)$ & $489(36.6)$ & 0.00 \\
\hline 2 & $751(23.7)$ & $598 \quad(19.3)$ & 0.11 & $228 \quad(17.0)$ & $237(17.7)$ & 0.01 \\
\hline $3+$ & $408(12.9)$ & $190 \quad(6.1)$ & 0.23 & $60 \quad(4.5)$ & $57 \quad(4.3)$ & 0.04 \\
\hline
\end{tabular}




\section{TABLE 1 Demographics and Baseline Clinical Characteristics of Study Patients (continued)}

n (\%)

\begin{tabular}{|c|c|c|c|c|c|}
\hline \multicolumn{3}{|c|}{ Before Matching } & \multicolumn{3}{|c|}{ After Matching } \\
\hline $\begin{array}{c}\text { Erenumab } \\
n=3,171\end{array}$ & $\begin{array}{l}\text { OnabotA } \\
n=3,100\end{array}$ & SMD & $\begin{array}{c}\text { Erenumab } \\
n=1,338\end{array}$ & $\begin{array}{c}\text { OnabotA } \\
n=1,338\end{array}$ & SMD \\
\hline $1,338 \quad(42.2)$ & $1,129 \quad(36.4)$ & 0.12 & $488 \quad(36.5)$ & $489 \quad(36.6)$ & 0.00 \\
\hline $920 \quad(29.0)$ & $826 \quad(26.6)$ & 0.05 & $318 \quad(23.8)$ & $322(24.1)$ & 0.01 \\
\hline $720 \quad(22.7)$ & 0 & 0.77 & 0 & 0 & 0.00 \\
\hline $597(18.8)$ & $532 \quad(17.2)$ & 0.04 & $214(16.0)$ & $213(15.9)$ & 0.00 \\
\hline $274 \quad(8.6)$ & $219 \quad(7.1)$ & 0.06 & $94 \quad(7.0)$ & $92 \quad(6.9)$ & 0.01 \\
\hline $61 \quad(1.9)$ & $47 \quad(1.5)$ & 0.03 & $13 \quad(1.0)$ & $20 \quad(1.5)$ & 0.05 \\
\hline
\end{tabular}

Preventive drugs used, 12-month pre-index

\begin{tabular}{l|r}
\hline Anticonvulsant & 1,33 \\
\hline Antidepressant & \\
\hline OnabotulinumtoxinA & \\
\hline Beta blocker & \\
\hline Calcium channel blocker & \\
\hline ACE inhibitor & 627 \\
\hline
\end{tabular}

Number of claims for preventive drugs (with migraine diagnosis), mean (SD)

\begin{tabular}{l|l|l|l|l|l|l}
\hline Anticonvulsant & $1.07(1.92)$ & $0.76(1.40)$ & 0.19 & $0.76(1.44)$ & $0.72(1.35)$ & 0.03 \\
\hline Antidepressant & $0.66(1.37)$ & $0.50(1.11)$ & 0.13 & $0.47(1.05)$ & $0.42(0.93)$ & 0.05 \\
\hline OnabotulinumtoxinA & $0.61(1.32)$ & 0 & 0.66 & 0 & 0 & 0.00 \\
\hline Beta blocker & $0.38(1.04)$ & $0.27(0.72)$ & 0.12 & $0.31(0.91)$ & $0.24(0.66)$ & 0.08 \\
\hline Calcium channel blocker & $0.18(0.77)$ & $0.12(0.51)$ & 0.10 & $0.13(0.60)$ & $0.12(0.53)$ & 0.02 \\
\hline ACE inhibitor & $0.04(0.32)$ & $0.02(0.19)$ & 0.06 & $0.02(0.24)$ & $0.02(0.14)$ & 0.01 \\
\hline
\end{tabular}

Number of acute drug classes used, 6-month pre-index, mean (SD)

\begin{tabular}{|c|c|c|c|c|c|c|}
\hline 0 & $872 \quad(27.5)$ & $1,256 \quad(40.5)$ & 0.27 & $568 \quad(42.5)$ & $568 \quad(42.5)$ & 0.00 \\
\hline 1 & $1,545 \quad(48.7)$ & $1,243 \quad(40.1)$ & 0.17 & $582(43.5)$ & 585 (43.7) & 0.00 \\
\hline 2 & $560 \quad(17.7)$ & $434 \quad(14.0)$ & 0.10 & $145(10.8)$ & 151 (11.3) & 0.01 \\
\hline $3+$ & $194 \quad(6.1)$ & $167 \quad(5.4)$ & 0.03 & $43 \quad(3.2)$ & $34 \quad(2.5)$ & 0.04 \\
\hline
\end{tabular}

Acute medication used, 6-month pre-index

\begin{tabular}{|c|c|c|c|c|c|c|}
\hline Any acute medication use & $2,247 \quad(70.9)$ & $1,776 \quad(57.3)$ & 0.14 & $741 \quad(55.4)$ & $742(55.5)$ & 0.00 \\
\hline Triptans & $1,774 \quad(55.9)$ & $1,395 \quad(45.0)$ & 0.07 & $578(43.2)$ & $578 \quad(43.2)$ & 0.00 \\
\hline Opioids & $625 \quad(19.7)$ & $449 \quad(14.5)$ & 0.14 & $180(13.5)$ & $171(12.8)$ & 0.02 \\
\hline NSAIDs & $236 \quad(7.4)$ & (7.0) & 0.02 & (4.4) & $64 \quad(4.8)$ & 0.02 \\
\hline Barbiturates & $211 \quad(6.7)$ & (7.1) & 0.02 & $(4.9)$ & $71 \quad(5.3)$ & 0.02 \\
\hline Ergots & $92 \quad(2.9)$ & $38 \quad(1.2)$ & 0.07 & $19 \quad(1.4)$ & $(0.9)$ & 0.05 \\
\hline
\end{tabular}

Number of claims for acute medication drugs, mean (SD)

\begin{tabular}{l|l|l|l|l|l|l|l}
\hline Any acute medication use & $3.29(4.40)$ & $2.20(3.68)$ & 0.13 & $2.16(3.14)$ & $2.23(3.65)$ & 0.02 \\
\hline Triptans & $2.63(4.05)$ & $1.72(3.42)$ & 0.08 & $1.81(2.96)$ & $1.86(3.42)$ & 0.02 \\
\hline Opioids & $0.40(1.26)$ & $0.27(0.87)$ & 0.12 & $0.21(0.64)$ & $0.22(0.75)$ & 0.01 \\
\hline NSAIDs & $0.10(0.39)$ & $0.08(0.33)$ & 0.03 & $0.05(0.26)$ & $0.05(0.26)$ & 0.01 \\
\hline Barbiturates & $0.10(0.45)$ & $0.10(0.42)$ & 0.00 & $0.07(0.36)$ & $0.08(0.37)$ & 0.02 \\
\hline Ergots & $0.07(0.54)$ & $0.03(0.29)$ & 0.07 & $0.02(0.25)$ & $0.02(0.18)$ & 0.04 \\
\hline
\end{tabular}




\section{TABLE 1 Demographics and Baseline Clinical Characteristics of Study Patients (continued)}

\begin{tabular}{|c|c|c|c|c|c|c|}
\hline \multirow[b]{2}{*}{$\mathbf{n}(\%)$} & \multicolumn{3}{|c|}{ Before Matching } & \multicolumn{3}{|c|}{ After Matching } \\
\hline & $\begin{array}{c}\text { Erenumab } \\
n=3,171\end{array}$ & $\begin{array}{l}\text { OnabotA } \\
n=3,100\end{array}$ & SMD & $\begin{array}{c}\text { Erenumab } \\
n=1,338\end{array}$ & $\begin{array}{l}\text { OnabotA } \\
n=1,338\end{array}$ & SMD \\
\hline \multicolumn{7}{|c|}{ Proportion of patients who used health care resources } \\
\hline \multicolumn{7}{|l|}{ ED/inpatient visits } \\
\hline All-cause & $1,096(34.6)$ & $1,092(35.2)$ & 0.01 & $401(30.0)$ & $471(35.2)$ & 0.11 \\
\hline Migraine-specific & $231 \quad(7.3)$ & $146 \quad(4.7)$ & 0.11 & $51 \quad(3.8)$ & $49 \quad(3.7)$ & 0.01 \\
\hline \multicolumn{7}{|l|}{ Office visits } \\
\hline All-cause & $3,062 \quad(96.6)$ & $2,919(94.2)$ & 0.11 & $1,285(96.0)$ & 1,275 (95.3) & 0.04 \\
\hline Migraine-specific & $2,873 \quad(90.6)$ & $2,588 \quad(83.5)$ & 0.21 & $1,180 \quad(88.2)$ & $1,156 \quad(86.4)$ & 0.05 \\
\hline \multicolumn{7}{|l|}{ Other outpatient visits } \\
\hline All-cause & $2,913 \quad(91.9)$ & $2,839(91.6)$ & 0.01 & $1,228 \quad(91.8)$ & $1,221 \quad(91.3)$ & 0.02 \\
\hline Migraine-specific & $1,141 \quad(36.0)$ & $1,118 \quad(36.1)$ & 0.00 & $422(31.5)$ & $430 \quad(32.1)$ & 0.01 \\
\hline \multicolumn{7}{|c|}{ Neurologist/headache specialist visits } \\
\hline All-cause & $2,545 \quad(80.3)$ & $2,441 \quad(78.7)$ & 0.04 & $1,070 \quad(80.0)$ & $1,039 \quad(77.7)$ & 0.06 \\
\hline Migraine-specific & $2,357 \quad(74.3)$ & $2,170 \quad(70.0)$ & 0.10 & $974(72.8)$ & $937(70.0)$ & 0.06 \\
\hline \multicolumn{7}{|c|}{ Number of claims for HCRU, mean (SD) } \\
\hline \multicolumn{7}{|l|}{ ED/inpatient visits } \\
\hline All-cause & $1.61(4.40)$ & $1.65(4.55)$ & 0.01 & $1.32(3.88)$ & $1.60(4.23)$ & 0.07 \\
\hline Migraine-specific & $0.18 \quad(0.91)$ & $0.10 \quad(0.71)$ & 0.09 & $0.09 \quad(0.62)$ & $0.09 \quad(0.69)$ & 0.00 \\
\hline \multicolumn{7}{|l|}{ Office visits } \\
\hline All-cause & $19.79(16.24)$ & $19.43(16.74)$ & 0.02 & $17.8(15.2)$ & $18.66(15.26)$ & 0.06 \\
\hline Migraine-specific & $4.59(4.54)$ & $3.31(3.64)$ & 0.31 & 3.18 (2.67) & 3.23 (3.10) & 0.02 \\
\hline \multicolumn{7}{|l|}{ Other outpatient visits } \\
\hline All-cause & $10.45(15.44)$ & $10.60(14.09)$ & 0.01 & $9.28(12.12)$ & $10.05(13.13)$ & 0.06 \\
\hline Migraine-specific & $1.16(4.91)$ & $0.87 \quad(2.18)$ & 0.08 & $0.72(1.67)$ & $0.81(2.04)$ & 0.05 \\
\hline \multicolumn{7}{|c|}{ Neurologist/headache specialist visits } \\
\hline All-cause & $3.85(4.21)$ & $3.28 \quad(4.10)$ & 0.14 & $3.20(3.86)$ & $3.25(4.20)$ & 0.01 \\
\hline Migraine-specific & $2.49(2.58)$ & $1.76(2.15)$ & 0.31 & $1.81(1.77)$ & 1.75 (1.89) & 0.03 \\
\hline
\end{tabular}

Note: Data are $n(\%)$ unless otherwise stated.

$A C E=$ angiotensin-converting enzyme; $C M=$ chronic migraine; $E D=$ emergency department; $H C P=$ health care professional; $H C R U=$ health care resource utilization; NSAID = nonsteroidal anti-inflammatory drug; onabot $A=$ onabotulinumtoxin $A ; S D=$ standard deviation; SMD =standardized mean difference.

online article). When the $P$ value was $<0.05$, groups were considered to be significantly different.

\section{Results}

An overview of the study design is shown in Supplementary Figure 1 (available in online article). Patients with at least 1 migraine diagnosis between May 1, 2017, and September 30, 2019, were identified from the Optum's deidentified CDM database. In total, 3,171 patients met the criteria for inclusion in the erenumab cohort and 3,100 for the onabotulinumtoxinA cohort (Figure 1). Following stratified PS matching, the number of patients included in the analyses was 1,338 for each cohort. Demographics and baseline clinical characteristics for the pre-matched and post-matched cohorts are shown in Table 1. Prevalence of the comorbidities included in the CCI are shown in Supplementary Table 1 (available in online article). In the post-matched data, the SMD was mainly kept below 0.1 , a threshold that has been recommended for declaring balance. ${ }^{42}$ 


\section{FIGURE 2 Number of Claims per Person for Acute Medication in the 6-Month Post-Index Period (PS-Matched Model)}

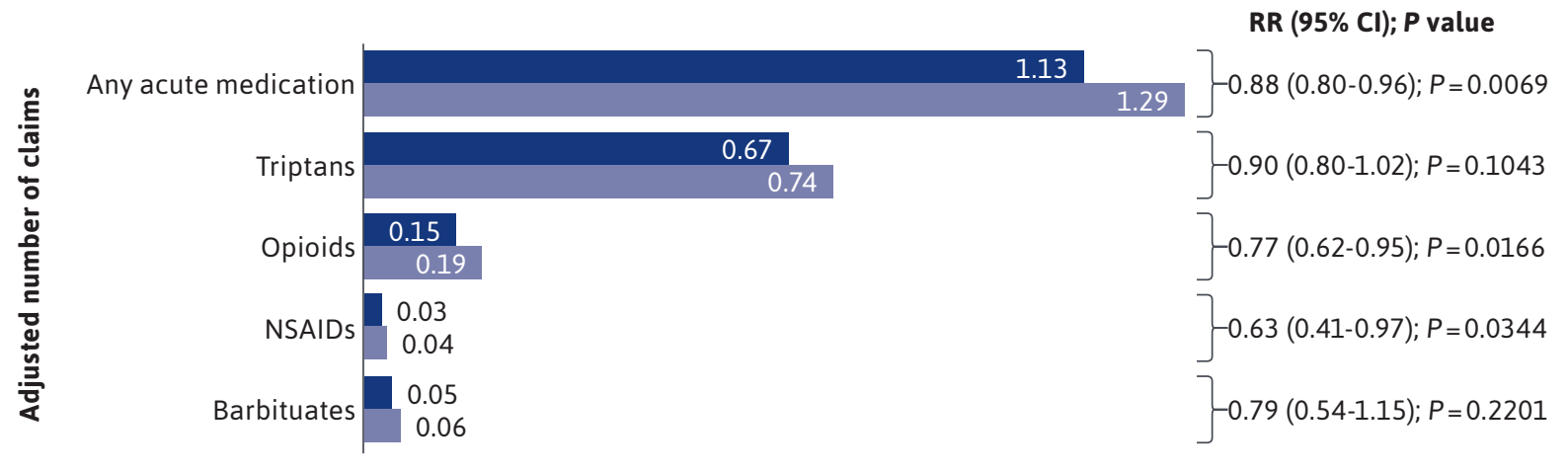

Erenumab $(n=1,338)$ OnabotulinumtoxinA $(n=1,338)$

Notes: Use of nonmigraine-specific acute medications (NSAIDs, opioids, and barbiturates) required a migraine diagnosis on or before 7 days of the medication claim. Negative binomial model with PS-matched data with covariate adjustment (age, gender, CCI, insurance type, region, selected comorbidities [CM, insomnia, depression, cardiovascular disease, irritable bowel syndrome, fibromyalgia], number of acute/preventive drugs by class [numerical], acute drug use by class [categorical], number of acute drugs [barbiturates, triptans, NSAIDs, opioids, antiemetics], all-cause inpatient or ED visits [binary], number of all-cause office visits, and number of all-cause office visits to a neurologist).

$C C l=C h a r l s o n$ Comorbidity Index; $C M=$ chronic migraine; $E D=$ emergency department; $N S A I D=$ nonsteroidal anti-inflammatory drug; $P S=$ propensity score; $R R=$ rate ratio.

\section{ACUTE MEDICATION USAGE IN THE 6-MONTH POST-INDEX PERIOD}

At 6 months, the adjusted average number of claims per person for any acute medication was significantly lower in the erenumab cohort (1.13 vs 1.29 in the onabotulinumtoxinA cohort; RR=0.88; 95\% CI=0.80-0.96; $\mathrm{P}=0.0069$; Figure 2); however, the adjusted proportion of patients with any acute medication use was similar for both cohorts (Supplementary Figure 2, available in online article).

The adjusted average number of claims per person for triptans in the 6-month post-index period was numerically lower, but statistically nonsignificant, in the erenumab cohort ( 0.67 vs 0.74 in the onabotulinumtoxin $\mathrm{A}$ cohort; RR=0.90; 95\% CI=0.80-1.02; $\mathrm{P}=0.1043$; Figure 2). The adjusted proportion of patients using triptans was numerically higher with erenumab $(46.1 \%$ vs $40.9 \%$ in the onabotulinumtoxinA cohort) but statistically nonsignificant $(\mathrm{OR}=1.24 ; 95 \% \mathrm{CI}=0.99-1.54 ; \mathrm{P}=0.0593$; Supplementary Figure 2). The adjusted average number of claims per person for opioids in the 6-month post-index period was significantly lower in the erenumab cohort ( 0.15 vs 0.19 in the onabotulinumtoxinA cohort; $\mathrm{RR}=0.77 ; 95 \% \mathrm{CI}=0.62-$ $0.95 ; \mathrm{P}=0.0166$; Figure 2). Similarly, the adjusted proportion of patients who used opioids was lower in the erenumab cohort $(10.9 \%$ vs $13.7 \%$ in the onabotulinumtoxinA cohort;
$\mathrm{OR}=0.77 ; 95 \% \mathrm{CI}=0.61-0.98 ; \mathrm{P}=0.0350 ;$ Supplementary Figure 2). The adjusted average number of claims per person for NSAIDs was also significantly lower in the erenumab cohort $(0.03$ vs 0.04 in the onabotulinumtoxinA cohort; $\mathrm{RR}=0.63 ; 95 \% \mathrm{CI}=0.41-0.97 ; \mathrm{P}=0.0344$; Figure 2 ), as was the adjusted proportion of patients who used NSAIDs in the 6 -month post-index period $(2.4 \%$ vs $3.6 \%$ in the onabotulinumtoxinA cohort; $\mathrm{OR}=0.65 ; 95 \% \mathrm{CI}=0.43-0.98 ; \mathrm{P}=0.0413$; Supplementary Figure 2). The adjusted number of claims per person for barbiturates was numerically lower, but statistically nonsignificant, in the erenumab cohort $(0.05$ vs 0.06 in the onabotulinumtoxinA cohort; RR $=0.79 ; 95 \%$ $\mathrm{CI}=0.54-1.15 ; \mathrm{P}=0.2201)$. Similarly, the adjusted proportion of patients who used barbiturates was numerically lower, but statistically nonsignificant, in the erenumab cohort (3.8\% vs $4.6 \%$ in the onabotulinumtoxinA cohort; OR=0.83; 95\% CI=0.58-1.19; $\mathrm{P}=0.3117)$. The results for ergot use in the 6 -month post-index period are not reported due to insufficient data.

The adjusted number of different types of acute medications used (ie, $0,1,2$, or $3^{+}$) at drug class level and generic drug level was similar for both cohorts (data not shown). Sensitivity analysis using the IPTW model demonstrated similar findings for the number of claims per person for acute medication use (Supplementary Figure 3) and for 


\section{FIGURE 3 Average Number of Visits per Person to Health Care Providers in the 6-Month Post-Index Period (PS-Matched Model)}

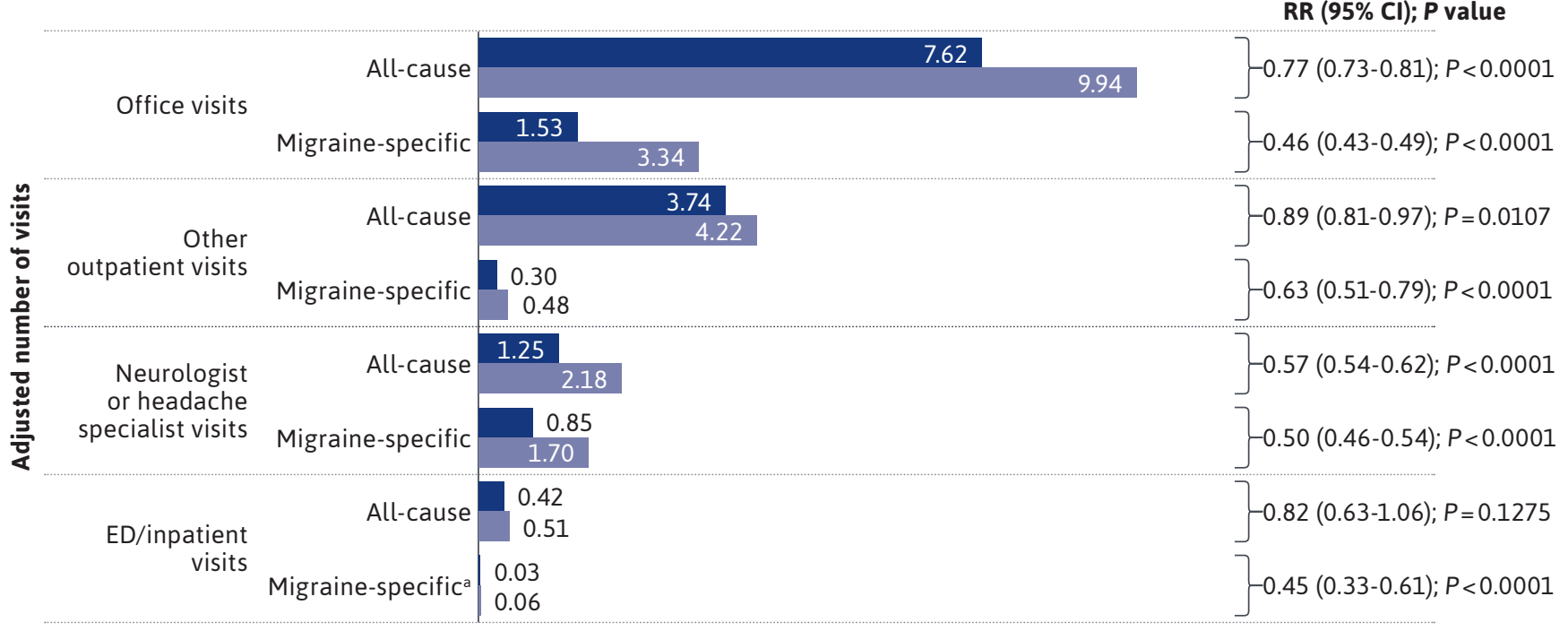

Erenumab $(\mathrm{n}=1,338)$ OnabotulinumtoxinA $(\mathrm{n}=1,338)$

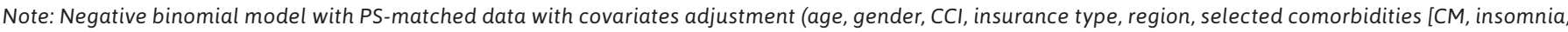
depression, cardiovascular disease, irritable bowel syndrome, fibromyalgia], number of acute/preventive drugs by class [numerical], acute drug use by class

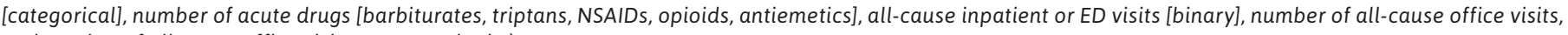
and number of all-cause office visits to a neurologist).

aPS-matched model did not converge (results for IPTW model are shown).

$\mathrm{CCl}=$ Charlson Comorbidity Index; $\mathrm{CM}=$ chronic migraine; $\mathrm{ED}=$ emergency department; IPTW=inverse probability of treatment weighting; NSAID=nonsteroidal anti-inflammatory drug; $P S=$ propensity score; $R R=$ rate ratio.

the proportion of patients who used acute medication (Supplementary Figure 4 , both figures available in online article) in the 6-month post-index period.

\section{HCRU IN THE 6-MONTH POST-INDEX PERIOD}

By 6 months, the adjusted average number of all-cause office visits per person was significantly lower in the erenumab cohort (7.62 vs 9.94 in the onabotulinumtoxinA cohort; RR=0.77; 95\% CI=0.73-0.81; $\mathrm{P}<0.0001$; Figure 3). Similarly, the adjusted average number of migrainespecific office visits per person was significantly lower in the erenumab cohort (1.53 vs 3.34 in the onabotulinumtoxinA cohort; $\mathrm{RR}=0.46 ; 95 \% \mathrm{CI}=0.43-0.49 ; \mathrm{P}<0.0001$; Figure 3). All patients in both cohorts had an all-cause office visit (data from IPTW model), but migraine-specific office visits were lower in the erenumab cohort $(79.9 \%$ vs $94.0 \%$ in the onabotulinumtoxinA cohort; OR $=0.26 ; 95 \%$ $\mathrm{CI}=0.21-0.31 ; \mathrm{P}<0.0001 ; \underline{\text { Supplementary Figure 5}}$, available in online article).
The adjusted average number of all-cause visits per person to a neurologist or headache specialist in the 6 -month post-index period was lower in the erenumab cohort (1.25 vs 2.18 in the onabotulinumtoxinA cohort; $\mathrm{RR}=0.57 ; 95 \% \mathrm{CI}=0.54-0.62 ; \mathrm{P}<0.0001$; Figure 3 ), as was the adjusted number of migraine-specific visits per person to a neurologist or headache specialist $(0.85$ vs 1.70 in the onabotulinumtoxinA cohort; $\mathrm{RR}=0.50 ; 95 \% \mathrm{CI}=0.46-0.54$; $\mathrm{P}<0.0001$; Figure 3 ). The adjusted proportion of patients with all-cause visits to a neurologist or headache specialist in the 6-month post-index period was $81.0 \%$ in the erenumab cohort compared with $90.9 \%$ in the onabotulinumtoxinA cohort $(\mathrm{OR}=0.43 ; 95 \% \mathrm{CI}=0.37-0.49 ; \mathrm{P}<0.0001)$ (data from IPTW model), while the adjusted proportion of patients with migraine-specific visits to a neurologist or headache specialist was $58.8 \%$ in the erenumab cohort compared with $77.4 \%$ in the onabotulinumtoxinA cohort $(\mathrm{OR}=0.41 ; 95 \% \mathrm{CI}=0.35-0.49 ; \mathrm{P}<0.0001 ;$ Supplementary Figure 5, available in online article). 


\section{FIGURE 4 Number of Composite Endpoint Events per Person in the 6-Month Post-Index Period (PS-Matched Model)}

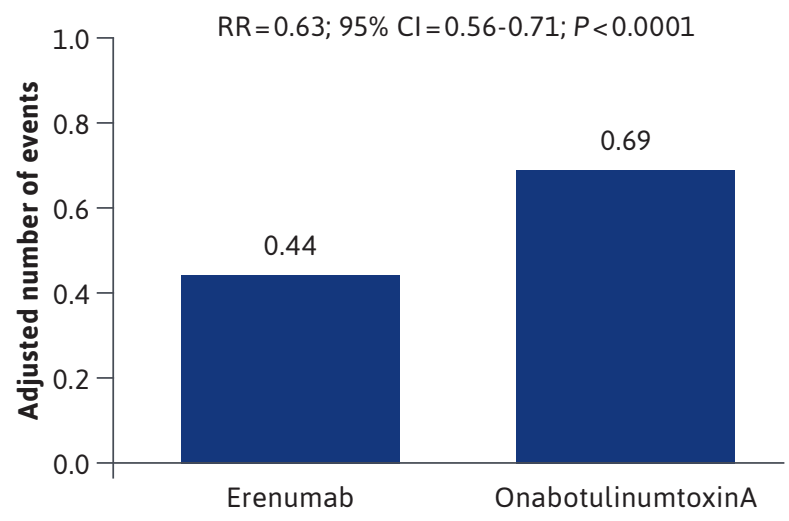

Notes: Composite endpoint of: (1) outpatient visit with a diagnosis of migraine and an associated acute medication claim, (2) hospital admission with a primary diagnosis for migraine, or (3) ED visit with a primary diagnosis for migraine.

Negative binomial model with PS-matched data with covariates adjustment (age, gender, $\mathrm{CCl}$, insurance type, region, selected comorbidities [CM, insomnia, depression, cardiovascular disease, irritable bowel syndrome, fibromyalgia), number of acute/preventive drugs use by class [numerical], acute drug use by class [categorical], number of acute drugs [barbiturates, triptans, NSAIDs, opioids, antiemetics], all-cause inpatient or ED visit (binary), number of all-cause office visits, and number of all-cause office visits to a neurologist).

$\mathrm{CCl}=$ Charlson Comorbidity Index; $\mathrm{CM}=$ chronic migraine; $\mathrm{ED}=$ emergency department; $P S=$ propensity score; $R R=$ rate ratio.

The adjusted average number of all-cause other outpatient visits per person in the 6 -month post-index period was lower in the erenumab cohort (3.74 vs 4.22 in the onabotulinumtoxinA cohort; $\mathrm{RR}=0.89 ; 95 \% \mathrm{CI}=0.81-0.97 ; \mathrm{P}=0.0107$ ), as was the adjusted number of migraine-specific other outpatient visits $(0.30$ vs 0.48 in the onabotulinumtoxinA cohort; $\mathrm{RR}=0.63 ; 95 \% \mathrm{CI}=0.51-0.79 ; \mathrm{P}<0.0001$; Figure 3 ). Similarly, fewer patients in the erenumab cohort had all-cause and migraine-specific other outpatient visits compared with the onabotulinumtoxinA cohort (Supplementary Figure 5, available in online article).

The adjusted average number of all-cause ED/inpatient visits per person in the 6-month post-index period was not significantly different between cohorts; however, the adjusted number of migraine-specific ED/inpatient visits was lower in the erenumab cohort $(0.03$ vs 0.06 in the onabotulinumtoxinA cohort; $\mathrm{RR}=0.45 ; 95 \% \mathrm{CI}=0.33-$ $0.61 ; \mathrm{P}<0.0001$ [data from IPTW model]; Figure 3$)$. In the 6 -month post-index period, significantly fewer patients in the erenumab cohort had an all-cause ED/inpatient visit
(17.5\%) compared with the onabotulinumtoxinA cohort (21.6\%) $(\mathrm{OR}=0.77 ; 95 \% \mathrm{CI}=0.63-0.94 ; \mathrm{P}=0.0105)$, while numerically fewer patients in the erenumab cohort had a migraine-specific ED/inpatient visit $(2.4 \%$ vs $3.3 \%$ in the onabotulinumtoxinA cohort; $\mathrm{OR}=0.71 ; 95 \% \mathrm{CI}=0.48-1.04$; $\mathrm{P}=0.0810 ;$ Supplementary Figure 5, available in online article).

Sensitivity analysis using the IPTW model demonstrated similar findings for the number of all-cause and migrainespecific visits to health care providers (Supplementary Figure 6) and for the proportion of patients with allcause and migraine-specific visits to health care providers (Supplementary Figure 7, both figures available in online article) in the 6-month post-index period.

\section{COMPOSITE ENDPOINT}

Patients in the erenumab cohort had a significantly lower number of composite events than the onabotulinumtoxinA cohort (0.44 vs 0.69; RR=0.63; 95\% CI=0.56-0.71; $\mathrm{P}<0.0001$; Figure 4). A lower proportion of patients with any of the 3 composite events was also observed in the erenumab cohort compared with the onabotulinumtoxinA cohort (31.7\% vs 44.3\%; OR=0.59; 95\% CI=0.49-0.70; $\mathrm{P}<0.0001$; Supplementary Figure 8, available in online article).

Sensitivity analysis using the IPTW model demonstrated similar findings for the number of composite events per person (Supplementary Figure 9) and for the proportion of patients with a composite event (Supplementary Figure 10, both figures available in online article) in the 6-month postindex period.

\section{Discussion}

Preventive treatments are an important part of the overall approach to migraine management for a large proportion of patients. ${ }^{8}$ Erenumab belongs to a new class of CGRP mAbs that were specifically designed for migraine prevention. ${ }^{43}$ Therefore, it is important to evaluate the impact of erenumab on acute medication usage and HCRU and to compare these findings with the relative impact of nonspecific migraine preventive therapies, such as onabotulinumtoxinA. In this assessment of commercially insured patients with migraine in the United States, we found that acute medication usage and HCRU were generally lower in patients treated with erenumab than with onabotulinumtoxinA in the 6-month post-index period.

The use of acute medication in the treatment of migraine is almost universal, with more than $90 \%$ of patients reporting some type of acute medication use in their treatment regimen. ${ }^{44}$ Despite this, their use is often associated with limited effectiveness and poor tolerability. ${ }^{11,12}$ Studies have 
shown that suboptimal treatment with acute medication can result in an increased likelihood of disease progression and associated disability, ${ }^{45,46}$ which in turn can lead to greater utilization of health care resources. ${ }^{47}$ Overuse of acute medication, which is associated with chronification, greater pain intensity, and development of $\mathrm{MOH},{ }^{16-18}$ is more likely to occur in triptan, opioid, and barbiturate users and may be less likely to occur in NSAID users. ${ }^{14}$

In this study, we found that the number of claims per person for any acute medication in the 6-month post-index period was lower in the erenumab cohort compared with the onabotulinumtoxinA cohort, while the proportion of patients who used any acute medication was similar for both cohorts. This suggests that while patients continued to use acute medication following treatment with erenumab or onabotulinumtoxinA, their frequency of use was lower with erenumab. One possible explanation is that erenumab improves response to acute treatment, in turn leading to a lower frequency of intake; however, further studies are required to confirm this.

Of particular note, the number of claims per person for opioids was significantly lower in the erenumab cohort in the 6-month post-index period compared with the onabotulinumtoxinA cohort. Furthermore, significantly fewer patients in the erenumab cohort used opioids during this time period. Opioids are not recommended for regular use in the treatment of migraine due to the risk of developing dependency and a host of poor comorbid medical and psychiatric outcomes. ${ }^{48,49}$ Use of opioids has also been associated with increased risk of EM transformation to CM and an increased risk of MOH. ${ }^{17,18}$ Despite safer options, opioids continue to be used in over $50 \%$ of migraine-related ED visits, ${ }^{50,51}$ and overuse of opioids is a major factor contributing to increased resource use in patients with migraine. ${ }^{48,52}$ Use of barbiturates, which is also not recommended for routine treatment of migraine,${ }^{17}$ was numerically lower, but statistically nonsignificant, in the erenumab cohort compared with the onabotulinumtoxinA cohort; in this case, the lack of statistical significance is likely due to the small number of barbiturate users included in the analyses.

Depending on the severity of attacks, triptans or NSAIDs are generally recommended as first-line options for the acute treatment of migraine. ${ }^{53}$ In this study, the number of claims per person for NSAIDs in the 6-month postindex period was significantly lower in the erenumab cohort compared with the onabotulinumtoxinA cohort; however, the number of claims for triptans was statistically nonsignificant. Together, these findings suggest that while erenumab can reduce the use of opioids and NSAIDs (and any acute medication when analyzed together) to a greater extent than onabotulinumtoxinA over a 6-month period, triptan and barbiturate use remains similar with both migraine preventive medications. Whether a longer duration of treatment (or a bigger sample size in the case of barbiturates) is needed to observe a difference between cohorts remains to be seen. A separate publication will provide a robust assessment of the effectiveness of erenumab on acute medication use and HCRU in patients with migraine 6 months before and after treatment initiation.

Migraine imposes a substantial direct and indirect cost burden on the United States. ${ }^{5}$ Health care and lost productivity costs associated with migraine are estimated at $\$ 36$ billion annually, ${ }^{1}$ with the majority of direct costs due to higher utilization of health care resources in migraine patients compared with demographically matched controls. ${ }^{5}$ Headache is consistently the fourth- or fifth-leading cause of ED visits ${ }^{54}$ with migraine prompting at least 1.2 million ED visits in the United States annually. ${ }^{55}$ Previous studies have shown that the use of preventive therapy alongside acute treatment can reduce resource utilization in patients with migraine..$^{19,56,57}$ In this study, patients in the erenumab cohort made significantly fewer all-cause and migraine-specific office visits compared with the onabotulinumtoxinA cohort. In particular, the number of migraine-specific visits to a neurologist or headache specialist, which may be indicative of greater disease severity, was lower in the erenumab cohort, as was the number of migraine-specific ED/inpatient visits. We recently demonstrated that outpatient visits, including office and other outpatient visits, are the main driver of HCRU-associated cost. ${ }^{58}$ Since erenumab reduced the number of these visits to a greater extent than onabotulinumtoxinA, HCRUassociated costs would also likely be lower with erenumab; however, further studies are needed to confirm this.

Composite endpoints such as the one reported here have the potential to be used as a proxy to evaluate migraine attacks, although further validation is warranted. In this study, the number of composite events per person and the proportion of patients with any of the 3 composite events in the 6-month post-index period were significantly lower in the erenumab cohort compared with the onabotulinumtoxinA cohort. These findings suggest that preventive treatment with erenumab may be associated with a greater reduction in the frequency of migraine attacks than treatment with onabotulinumtoxinA, which in turn could lead to an overall reduction in the burden of the disease.

In clinical practice, onabotulinumtoxinA is approved for use in CM only, while erenumab can be used for the preventive treatment of patients with EM and CM. ${ }^{25,29}$ By using PS-matched cohorts, any inherent differences between 
these treatment groups are removed. Furthermore, a sensitivity analysis using the IPTW model produced similar findings.

\section{LIMITATIONS}

Using retrospective data from administrative claims has several limitations that should be noted. Due to the observational design of the study, the analysis may be affected by unobserved confounding factorseg, data contained in administrative claims databases may be inaccurate or subject to human or technical error. US claims data are also dependent on professional International Classification of Diseases coding, not use of the International Classification of Headache Disorders, 3rd Edition; therefore, some diagnoses may be missed, different professional types may have different coding patterns, and not all coding may be accurate.

Clinical information is also lacking beyond diagnosis codes. Optum's deidentified CDM database only includes data on insured patients in the United States; therefore, study findings are not generalizable to the entire population. Furthermore, patients with migraine frequently use over-thecounter acute medication, which is generally not included in the claims database and is thus unaccounted for in this study.

Patients receiving erenumab from the free drug program are also not captured in this database; thus, it is impossible to ascertain whether the first erenumab claim is truly indicative of the first time erenumab is used by a given patient.

Another potential limitation is that onabotulinumtoxinA has a quarterly dosing regimen administered by a health care professional, while erenumab is self-administered by the patient. Although onabotulinumtoxinA users may have had at least 1 more office visit than erenumab users during the 6-month follow-up time period in order to facilitate onabotulinumtoxinA administration, it is likely physicians required a follow-up visit with erenumab users within 3 months of treatment initiation since this was a relatively new biologic from a new drug class at the time.

Finally, it should be noted that the absence of an event, condition, or medication in the database is not confirmatory that it did not occur, while prescriptions filled at a pharmacy are not confirmatory of the medication being consumed/self-administered by the patient.

\section{Conclusions}

The findings from this retrospective real-world study indicate that erenumab reduces the use of acute medications (opioids and NSAIDs; any acute medication when analyzed together) to a greater extent than onabotulinumtoxinA. Furthermore, erenumab was associated with lower HCRU and a lower number of composite events. Together, these findings suggest erenumab may be more effective as a migraine preventive therapy in this study population during this period of time.

\section{DISCLOSURES}

This study was supported by Novartis Pharma AG. Novartis employees contributed to the study design, analysis of the data, and the decision to publish the results. Fang, Abdrabboh, Glassberg, Vo, and Ferraris are employed by Novartis. Zhou and Shen are employed by KMK Consulting, Inc., which received funding from Novartis to conduct the study.

Tepper reports grants from Allergan, Amgen, ElectroCore, Eli Lilly, Lundbeck, Neurolief, Novartis, Satsuma, and Zosano, outside the submitted work; personal fees from Dartmouth-Hitchcock Medical Center, American Headache Society, Thomas Jefferson University, Aeon, Align Strategies, Allergan/AbbVie, Alphasights, Amgen, Aperture Venture Partners,
Aralez Pharmaceuticals Canada, Axsome Therapeutics, Becker Pharmaceutical Consulting, BioDelivery Sciences International, Biohaven, ClearView Healthcare Partners, CoolTech, CRG, Currax, Decision Resources, DeepBench, DRG, Eli Lilly, Equinox, ExpertConnect, GLG, Guidepoint Global Healthcare Consultancy Group Health Science Communications, HMP Communications, Impel, InteractiveForums, M3 Global Research, Magellan Rx Management, Medicxi, Navigant Consulting, Neurorelief, Nordic BioTech, Novartis Pulmatrix, Reckner Healthcare, Relevale, SAI MedPartners, Satsuma, Slingshot Insights, Spherix Global Insights, Sudler and Hennessey, Synapse Medical Communications, System Analytic, Teva, Theranica, Thought Leader Select, Trinity Partners, XOC, Zosano, Krog and Partners, and Lundbeck, outside the submitted work; and CME honoraria from American Academy of Neurology, American Headache Society, Cleveland Clinic Foundation, Diamond Headache Clinic, Elsevier, Forefront Collaborative, Hamilton General Hospital, Ontario, Canada, Headache Cooperative of New England, Henry Ford Hospital, Detroit, Inova, Medical Learning Institute PeerView, Medical Education Speakers Network, Miller Medical Communications, North American Center for CME, Physicians' Education Resource, Rockpointe, ScientiaCME, WebMD/Medscape.

The abstract and poster of these results were presented at The Migraine Trust Virtual Symposium (MTIS), October 3-9, 2020 .

\section{ACKNOWLEDGMENTS}

The authors thank Janis Noonan for providing medical writing support, which was funded by Novartis.

\section{REFERENCES}

1. Migraine Research Foundation. Migraine facts. Accessed December 14 2020. https://migraineresearchfoundation.org/about-migraine/migraine-facts/

2. Headache Classification Committee of the International Headache Society. The International Classification of Headache Disorders, 3rd edition (beta version). Cephalalgia. 2013;33(9):629-808. 
3. GBD 2019 Diseases and Injuries Collaborators. Global burden of 369 diseases and injuries in 204 countries and territories, 1990-2019: a systematic analysis for the Global Burden of Disease Study 2019. Lancet. 2020;396(10258):1204-22.

4. Hawkins K, Wang S, Rupnow M. Direct cost burden among insured US employees with migraine. Headache. 2008;48(4):553-63.

5. Bonafede M, Sapra S, Shah N, Tepper S, Cappell K, Desai P. Direct and indirect healthcare resource utilization and costs among migraine patients in the United States. Headache. 2018;58(5):700-14.

6. Bonafede M, Cai Q, Cappell K, et al. Factors associated with direct health care costs among patients with migraine. $J$ Manag Care Spec Pharm. 2017;23(11):11691176. doi: 10.18553/jmcp.2017.23.11.1169

7. D'Amico D, Tepper SJ. Prophylaxis of migraine: general principles and patient acceptance. Neuropsychiatr Dis Treat. 2008;4(6):1155-67.

8. American Headache Society. The American Headache Society position statement on integrating new migraine treatments into clinical practice. Headache. 2019;59(1):1-18.

9. Tepper SJ. Acute treatment of migraine. Neurol Clin. 2019;37(4):727-42.

10. Minen MT, Lindberg K, Wells RE, et al. Survey of opioid and barbiturate prescriptions in patients attending a tertiary care headache center. Headache. 2015;55(9):1183-91.

11. Buse DC, Pearlman SH, Reed ML, Serrano D, Ng-Mak DS, Lipton RB. Opioid use and dependence among persons with migraine: results of the AMPP study. Headache. 2012;52(1):18-36.

12. Leroux E, Buchanan A, Lombard L, et al. Evaluation of patients with insufficient efficacy and/or tolerability to triptans for the acute treatment of migraine: a systematic literature review. Adv Ther. 2020;37(12):4765-96.
13. Tepper SJ, Diener HC, Ashina M, et al. Erenumab in chronic migraine with medication overuse: subgroup analysis of a randomized trial. Neurology. 2019;92(20):e2309-20.

14. Schwedt TJ, Alam A, Reed ML, et al. Factors associated with acute medication overuse in people with migraine: results from the 2017 migraine in America symptoms and treatment (MAST) study. J Headache Pain. 2018;19(1):38.

15. Peters GA, Horton BT. Headache; with special reference to the excessive use of ergotamine tartrate and dihydroergotamine. J Lab Clin Med. 1950;36(6):972-73.

16. Bigal ME, Rapoport AM, Sheftell FD, Tepper SJ, Lipton RB. Transformed migraine and medication overuse in a tertiary headache centre-clinical characteristics and treatment outcomes. Cephalalgia. 2004;24(6):483-90.

17. Bigal ME, Serrano D, Buse D, Scher A, Stewart WF, Lipton RB. Acute migraine medications and evolution from episodic to chronic migraine: a longitudinal population-based study. Headache. 2008;48(8):1157-68.

18. Fischer MA, Jan A. Medicationoveruse headache. Updated June 30, 2020. Accessed December 14, 2020. https://www.ncbi.nlm.nih.gov/books/ NBK538150/

19. Silberstein SD. Preventive migraine treatment. Continuum (Minneap Minn). 2015;21(4 Headache):973-89.

20. Estemalik E, Tepper S. Preventive treatment in migraine and the new US guidelines. Neuropsychiatr Dis Treat. 2013;9:709-20.

21. Sprenger T, Viana M, Tassorelli C. Current prophylactic medications for migraine and their potential mechanisms of action. Neurotherapeutics. 2018;15(2):313-23.

22. Blumenfeld AM, Bloudek LM, Becker WJ, et al. Patterns of use and reasons for discontinuation of prophylactic medications for episodic migraine and chronic migraine: results from the second international burden of migraine study (IBMS-II). Headache. 2013;53(4):644-55.
23. Hepp Z, Bloudek LM, Varon SF. Systematic review of migraine prophylaxis adherence and persistence. J Manag Care Pharm. 2014;20(1):22-33. doi: 10.18553/ jmcp.2014.20.1.22

24. Hepp Z, Dodick DW, Varon SF, et al. Persistence and switching patterns of oral migraine prophylactic medications among patients with chronic migraine: a retrospective claims analysis. Cephalalgia. 2017;37(5):470-85.

25. Botox. Prescribing information. Allergan; October 2010. Accessed December 14, 2020. https://www. accessdata.fda.gov/drugsatfda_docs/ label/2011/103000s5236lbl.pdf

26. Dodick DW, Turkel CC, DeGryse RE, et al. OnabotulinumtoxinA for treatment of chronic migraine: pooled results from the double-blind, randomized, placebocontrolled phases of the PREEMPT clinical program. Headache. 2010;50(6):921-36.

27. Aurora SK, Dodick DW, Turkel CC, et al. OnabotulinumtoxinA for treatment of chronic migraine: results from the double-blind, randomized, placebo-controlled phase of the PREEMPT 1 trial. Cephalalgia. 2010;30(7):793-803.

28. Diener HC, Dodick DW, Aurora SK, et al. OnabotulinumtoxinA for treatment of chronic migraine: results from the double-blind, randomized, placebocontrolled phase of the PREEMPT 2 trial. Cephalalgia. 2010;30(7):804-14.

29. Aimovig. Prescribing information. Amgen; May 2018. Accessed December 14, 2020. https://www.accessdata.fda.gov/drugsatfda_docs/ label/2018/761077s000lbl.pdf

30. Emgality. Prescribing information. Eli Lilly and Company; September 2018. Accessed December 14, 2020. https:// www.accessdata.fda.gov/drugsatfda_ docs/label/2018/761063s000lbl.pdf

31. Ajovy. Prescribing information. Teva Pharmaceuticals USA, Inc.; September 2018. Accessed December 14, 2020. https://www.accessdata.fda. gov/drugsatfda docs/label/2018/ 761089s000lbl.pdf 
32. Vyepti. Prescribing information. Lundbeck Seattle BioPharmaceuticals, Inc.; February 2020. Accessed December 14, 2020. https://www. accessdata.fda.gov/drugsatfda_docs/ label/2020/761119s000lbl.pdf

33. Goadsby PJ, Reuter U, Hallstrom Y, et al. A controlled trial of erenumab for episodic migraine. N Engl J Med. 2017;377(22):2123-32.

34. Tepper S, Ashina M, Reuter U, et al. Safety and efficacy of erenumab for preventive treatment of chronic migraine: a randomised, double-blind, placebocontrolled phase 2 trial. Lancet Neurol. 2017;16(6):425-34.

35. Silberstein SD, Dodick DW, Bigal ME, et al. Fremanezumab for the preventive treatment of chronic migraine. N Engl J Med. 2017;377(22):2113-22.

36. Detke HC, Goadsby PJ, Wang S, Friedman DI, Selzler KJ, Aurora SK. Galcanezumab in chronic migraine: the randomized, double-blind, placebocontrolled REGAIN study. Neurology. 2018;91(24):e2211-21.

37. Lipton RB, Goadsby PJ, Smith J, et al. Efficacy and safety of eptinezumab in patients with chronic migraine: PROMISE-2. Neurology. 2020;94(13): e1365-77.

38. Barbanti P, Aurilia C, Fofi L, Egeo G, Ferroni P. The role of anti-CGRP antibodies in the pathophysiology of primary headaches. Neurol Sci. 2017;38(Suppl 1): 31-35.

39. Dodick DW, Ashina M, Brandes JL, et al. ARISE: A phase 3 randomized trial of erenumab for episodic migraine. Cephalalgia. 2018;38(6):1026-37.

40. Reuter U, Goadsby PJ, Lanteri-Minet M, et al. Efficacy and tolerability of erenumab in patients with episodic migraine in whom two-to-four previous preventive treatments were unsuccessful: a randomised, double-blind, placebocontrolled, phase 3b study. Lancet. 2018;392(10161):2280-87.
41. Vandenbroucke JP, von Elm E, Altman DG, et al. Strengthening the Reporting of Observational Studies in Epidemiology (STROBE): explanation and elaboration. Int J Surg. 2014;12(12):1500-24.

42. Stuart EA, Lee BK, Leacy FP.

Prognostic score-based balance measures can be a useful diagnostic for propensity score methods in comparative effectiveness research. J Clin Epidemiol. 2013;66 (8 Suppl):S84-90, e81.

43. Tso AR, Goadsby PJ. Anti-CGRP monoclonal antibodies: the next era of migraine prevention? Curr Treat Options Neurol. 2017;19(8):27.

44. Diamond S, Bigal ME, Silberstein S, Loder E, Reed M, Lipton RB. Patterns of diagnosis and acute and preventive treatment for migraine in the United States: results from the American Migraine Prevalence and Prevention study. Headache. 2007;47(3):355-63.

45. Lipton RB, Fanning KM, Serrano D, Reed ML, Cady R, Buse DC. Ineffective acute treatment of episodic migraine is associated with new-onset chronic migraine. Neurology. 2015;84(7):688-95.

46. Lipton RB, Hutchinson S, Ailani J, et al. Discontinuation of acute prescription medication for migraine: results from the Chronic Migraine Epidemiology and Outcomes (CaMEO) study. Headache. 2019;59(10):1762-72.

47. Silberstein SD, Lee L, Gandhi K, Fitzgerald T, Bell J, Cohen JM. Health care resource utilization and migraine disability along the migraine continuum among patients treated for migraine. Headache. 2018;58(10):1579-92.

48. Tepper SJ. Opioids should not be used in migraine. Headache. 2012;52(Suppl 1): 30-34.

49. Casucci G, Cevoli S. Controversies in migraine treatment: opioids should be avoided. Neurol Sci. 201334(Suppl 1): S125-28.
50. Minen MT, Tanev K, Friedman BW. Evaluation and treatment of migraine in the emergency department: a review. Headache. 2014;54(7):1131-45.

51. Dodson H, Bhula J, Eriksson S, Nguyen K. Migraine treatment in the emergency department: alternatives to opioids and their effectiveness in relieving migraines and reducing treatment times. Cureus. 2018;10(4):e2439.

52. Muzina DJ, Chen W, Bowlin SJ. A large pharmacy claims-based descriptive analysis of patients with migraine and associated pharmacologic treatment patterns. Neuropsychiatr Dis Treat. 2011;7:663-72.

53. Ong JJY, De Felice M. Migraine treatment: Current acute medications and their potential mechanisms of action. Neurotherapeutics. 2018;15(2):274-290.

54. Burch R, Rizzoli P, Loder E. The prevalence and impact of migraine and severe headache in the United States: figures and trends from government health studies. Headache. 2018;58(4):496-505.

55. Friedman BW, West J, Vinson DR, Minen MT, Restivo A, Gallagher EJ. Current management of migraine in US emergency departments: an analysis of the National Hospital Ambulatory Medical Care Survey. Cephalalgia. 2015;35(4):301-09.

56. Silberstein SD, Winner PK, Chmiel JJ. Migraine preventive medication reduces resource utilization. Headache. 2003;43(3):171-178.

57. Freitag FG, Lyss H, Nissan GR. Migraine disability, healthcare utilization, and expenditures following treatment in a tertiary headache center. Proc (Bayl Univ Med Cent). 2013;26(4):363-67.

58. Newman L, Vo P, Zhou L, et al. Healthcare utilization and costs in patients with migraine who have failed previous preventive treatments. Neurol Clin Pract. Published online April 1, 2021. doi.https://doi.org/10.1212/ CPJ.0000000000001076 